\title{
Identification et caractérisation de souches industrielles du genre Bifidobacterium par 2 méthodes de taxonomie moléculaire
}

\author{
C Herreman, D Asselin, G Novel
}

Laboratoire de génétique microbienne, IRBA, Université de Caen, 14032 Caen Cedex, France

(Reçu le 17 décembre 1993; accepté le 2 septembre 1994)

\begin{abstract}
Résumé - L'absence d'un système d'identification simple et rapide des espèces du genre Bifidobacterium rend difficile l'utilisation de ces bactéries par l'industrie laitière. En vue d'obtenir de bons critères discriminants, nous avons identifié et caractérisé 3 souches de ferments industriels par 2 méthodes de taxonomie moléculaire en les comparant avec les souches type des espèces de Bifidobacterium utilisées commercialement. Par hybridation ADN-ADN, nous avons confirmé l'identification d'une des souches à l'espèce $B$ longum, tandis que les 2 autres, supposées appartenir aux espèces $B$ infantis et $B$ bifidum, ont été identifiées à l'espèce $B$ animalis. De plus, le polymorphisme de restriction des ADN digérés par l'endonucléase Xbal et observés après électrophorèse en champs pulsés permet de distinguer ces souches entre elles.
\end{abstract}

Bifidobacterium / taxonomie / polymorphisme des fragments de restriction / électrophorèse en champs pulsés / hybridation ADN-ADN

Summary - Identification and characterization of Bifidobacterium industrial strains by methods of molecular taxonomy. The lack of a simple and rapid identification system for species of the Bifidobacterium genus is a difficulty in the use of these bacteria in industrial transformation. To obtain valuable discriminating criteria, we have identified and characterized three industrial starter strains by two methods of molecular taxonomy by comparing with type strains of presumed commercially used species of Bifidobacterium. By DNA-DNA hybridization we confirmed the identification of one strain to Bifidobacterium longum species and of two others, presumed to belong to $\mathrm{B}$ infantis and $\mathrm{B}$ bifidum species, to Bifidobacterium animalis. DNA restriction $\mathrm{Xbal}$ fragment polymorphism revealed by pulsed field gel electrophoresis permitted to distinguish these strains.

Bifidobacterium / taxonomy / DNA restriction fragment polymorphism / pulsed-field gel electrophoresis / DNA-DNA hybridization 


\section{INTRODUCTION}

Depuis moins de 10 ans, les bifidobactéries sont utilisées dans la fabrication et la commercialisation de laits fermentés pour l'alimentation de l'homme. Plusieurs espèces de ce genre, celles notamment d'origine humaine, sont considérées comme des probiotiques, c'est-à-dire comme des micro-organismes qui transitent vivants dans le tube digestif à des concentrations suffisantes pour exprimer chez l'hôte certaines activités métaboliques (Fuller, 1992). Bien que les bénéfices de ces produits sur la santé humaine soient encore mal connus, les laits fermentés dits au "Bifidus actif» bénéficient d'une image de marque exceptionnellement bonne, ce qui a favorisé un développement considérable de ces produits sur le marché des produits frais.

Afin de contrôler l'effet probiotique des bifidobactéries, il est aujourd'hui nécessaire pour l'industriel d'identifier avec précision les espèces utilisées. En effet, les 28 espèces du genre Bifidobacterium ne sont pas toutes des organismes commensaux. L'espèce $B$ dentium est même considérée comme potentiellement pathogène (Gavini, 1989). Or, à ce jour l'identification et la taxonomie du genre Bifidobacterium par les méthodes "classiques» (profils fermentaire et enzymatique) soulèvent encore un certain nombre de problèmes, en particulier au niveau de la reconnaissance des espèces (Bahaka et al, 1993).

Dans cette étude, nous avons identifié et caractérisé, par 2 méthodes de taxonomie moléculaire, 3 souches industrielles du genre Bifidobacterium et nous avons comparé les résultats à ceux obtenus avec les souches type des espèces présumées.

\section{MATÉRIELS ET MÉTHODES}

\section{Souches et cultures}

Les souches testées, BL, BI, BB, proviennent de ferments industriels dits de "souches pures" et appartiennent, selon le producteur, respectivement aux 3 espèces d'origine humaine : $B$ longum, $B$ infantis et $B$ bifidum. L'origine des souches type utilisées dans cette étude est référencée dans le tableau $\mathrm{I}$.

\section{Hybridation ADN-ADN en milieu liquide}

La méthode d'hybridation quantitative choisie est une adaptation de la méthode de Crosa et al (1973). Il s'agit de l'hybridation en milieu liquide utilisant la nucléase $S_{1}$ suivie de la précipitation à l'acide trichloroacétique.

Tableau I. Souches type de Bifidobacterium utilisées dans cette étude.

Type strains of Bifidobacterium used in this work.

$\begin{array}{ll}\text { B longum } & \text { ATCC } 15707^{\top} \\ B \text { infantis } & \text { ATCC } 15697^{\top} \\ B \text { bifidum } & \text { ATCC } 29521^{\top} \\ B \text { breve } & \text { ATCC } 15700^{\top} \\ B \text { animalis } & \text { ATCC } 25527^{\top}\end{array}$

NCTC $11818^{\top}$ (Reuter E 194b)*

DSM $20088^{\top}$ (Reuter S12)*

NCFB $2242^{\top}$ (Mitsuoka R101-8)*

ATCC : American Type Culture Collection, Rockville, MD ; DSM : Deutsche Sammlung von Mikroorganismen, Göttingen, Germany ; NCFB : National Collection of Food Bacteria, Shinfield, Reading, UK; NCTC : National Collection of Type Cultures, Central Public Health Laboratory, London, United Kingdom. "Origine : Mme F Gavini, Institut national de la recherche agronomique, domaine du CERTIA, 59650 Villeneuve-d'Ascq Cedex, France 


\section{Électrophorèse en champs pulsés}

\section{Préparation de l'ADN chromosomique}

A partir d'une préculture de la nuit, $5 \mathrm{ml}$ de milieu frais sont ensemencés à $2 \%(v / v)$. Dès que la densité optique à $600 \mathrm{~nm}$ atteint $0,7-0,8$, les cellules sont traitées avec $1 \mu \mathrm{g} / \mathrm{ml}$ d'ampicilline durant $1 \mathrm{~h}$ à $37^{\circ} \mathrm{C}$ et centrifugées à $5000 \mathrm{~g}$ pendant $10 \mathrm{~min}$. Le culot cellulaire est lavé 2 fois avec $2,5 \mathrm{ml}$ de TEE (Tris-acétate $10^{-2} \mathrm{~mol} / \mathrm{I}$, EDTA $0,1 \mathrm{~mol} / \mathrm{l}, \mathrm{EGTA} 10^{-2} \mathrm{~mol} / \mathrm{p} \mathrm{pH}$ 8), et homogénéisé dans $300 \mu \mathrm{l}$ de TEE supplémenté en saccharose $(6,7 \% \mathrm{p} / \mathrm{v})$. La suspension cellulaire est alors ajoutée volume à volume à une solution d'agarose low melting (Nu Sieve GTG, FMC) à $3 \%$ dans du TAE (Tris $0,04 \mathrm{~mol} / \mathrm{l}$, acétate de sodium $0,04 \mathrm{~mol} / \mathrm{l}$, EDTA $\left.2.10^{-3} \mathrm{~mol} / \mathrm{l}\right)$ maintenue en surfusion à $42^{\circ} \mathrm{C}$.

\section{Lyse cellulaire}

Les blocs d'agarose sont placés dans $5 \mathrm{ml}$ de TEE et traités avec $25 \mathrm{mg}$ de lysozyme et $50 \mu \mathrm{l}$ de Sarkosyl $5 \%$ pendant $16 \mathrm{~h}$ à $37^{\circ} \mathrm{C}$ sous agitation. Après $16 \mathrm{~h}$ d'incubation à $37^{\circ} \mathrm{C}$ dans $5 \mathrm{ml}$ de TEE frais contenant $5 \mathrm{mg}$ de protéinase $\mathrm{K}$ et 500 $\mu \mathrm{g}$ de SDS $10 \%$, ces blocs sont dialysés dans $50 \mu \mathrm{l}$ de TE (Tris-acétate $10^{-2} \mathrm{~mol} / \mathrm{l}$, EDTA $10^{-3}$ $\mathrm{mol} / \mathrm{l}$ ) avec $200 \mu \mathrm{l}$ de PMSF (phényl méthane sulfanyl fluorure, $10^{-3} \mathrm{~mol} / \mathrm{l}$ ) pendant $1 \mathrm{~h} \mathrm{à} 4^{\circ} \mathrm{C}$ sous agitation douce, puis dans du TE à $4^{\circ} \mathrm{C}$ pendant $1 \mathrm{~h}, 2$ fois de suite.

\section{Digestion enzymatique}

Les ADN sont ensuite hydrolysés par l'endonucléase de restriction Xbal, dejà utilisée par Pietersen (1991) et Bourget et al (1992) pour l'étude du génome des bifidobactéries. Chaque bloc est lavé pendant $15 \mathrm{~min}$ dans $1 \mathrm{ml}$ de tampon de digestion (Tris- $\mathrm{HCl} 5.10^{-2} \mathrm{~mol} / \mathrm{l}, \mathrm{MgCl}_{2}$ $10^{-2} \mathrm{~mol} / \mathrm{l}, \mathrm{NaCl} 0,1 \mathrm{~mol} / \mathrm{l}$, dithioérythritol $10^{-3}$ $\mathrm{mol} / \mathrm{l}, \mathrm{pH} 7,5$ ), puis incubé $16 \mathrm{~h}$ dans $200 \mu \mathrm{l}$ de ce tampon additionné de $2 \mu$ d'enzyme ( 24 unités) à $37^{\circ} \mathrm{C}$. La réaction est arrêtée avec $1 \mathrm{ml}$ de TEE.

\section{Électrophorèse en champs pulsés}

L'électrophorèse en champs pulsés (Tanskanen et al, 1990) est mise en cuvre à partir du système CHEF DR II (Biorad). Le gradient d'impul- sion utilisé est de 1 à 20 s pendant $20 \mathrm{~h}$ à $14^{\circ} \mathrm{C}$ et $180 \mathrm{~V}$.

\section{Analyse d'images}

Les négatifs photographiques des gels sont traités à l'aide de l'application Whole Band du logiciel Bio-image (Millipore) sur un ordinateur compatible PC.

\section{RÉSULTATS ET DISCUSSION}

\section{Hybridation ADN-ADN en milieu liquide}

Afin d'identifier avec certitude "l'espèce génomique" à laquelle appartiennent les 3 souches industrielles, nous avons utilisé la méthode d'hybridation quantitative ADNADN en milieu liquide (tableau II). Lorsque le pourcentage d'homologie obtenu entre la souche testée et la souche type était inférieur à $60 \%$, nous avons poursuivi l'identification avec d'autres souches type. Pour un pourcentage d'homologie compris entre $60 \%$ et $100 \%$, nous avons déterminé la différence de température de demi-dénaturation $(\Delta \mathrm{Tm})$ entre ces hybrides (selon la méthode de Grimont, 1988).

Selon le comité ad hoc de conciliation des approches systématiques bactériennes de 1987 (Wayne et al, 1987) l'appartenance d'une souche à une espèce donnée est déterminée par un pourcentage d'homologie d'ADN supérieur à $70 \%$ entre la souche testée et la souche type correspondante et une valeur $\Delta \mathrm{Tm}$ au plus égale à $5^{\circ} \mathrm{C}$.

\section{Les espèces $B$ longum et $B$ infantis}

Le pourcentage d'homologie élevé (127\%) obtenu après hybridation entre la souche $B L$ et la sonde $B$ longum NCTC $11818^{\top}$ mais aussi une $\triangle D T m$ entre ces 2 souches inférieure à $1,5^{\circ} \mathrm{C}$, nous permettent d'identifier BL à l'espèce $B$ longum. Toutefois, la 
Tableau II. Hybridation ADN-ADN en milieu liquide * (\% homologie). DNA-DNA hybridization in liquid medium (homology percentage).

\begin{tabular}{|c|c|c|c|c|c|c|}
\hline \multirow[b]{2}{*}{ Souches testées } & \multirow[b]{2}{*}{ s Souches } & \multicolumn{5}{|c|}{ Sonde } \\
\hline & & $\begin{array}{c}\text { B longum } \\
\text { NCTC 11818 }\end{array}$ & $\begin{array}{c}\text { B infantis } \\
D S M 20088^{T}\end{array}$ & $\begin{array}{c}\text { B bifidum } \\
\text { ATCC } 29521^{T}\end{array}$ & $\begin{array}{c}\text { B breve } \\
\text { ATCC15700T }\end{array}$ & $\begin{array}{l}B \text { animalis } \\
\text { NCFB } 2242^{T}\end{array}$ \\
\hline B longum & $\begin{array}{l}\text { NTC } 11818^{\top} \\
\text { ATCC } 15707^{\top}\end{array}$ & $\begin{array}{l}100 \\
132\end{array}$ & 69 & 9 & 38 & 13 \\
\hline$B$ infantis & $\begin{array}{l}\text { DSM 20088 } \\
\text { ATCC } 15697^{\top}\end{array}$ & $\begin{array}{l}61 \\
69\end{array}$ & $\begin{array}{l}100 \\
120\end{array}$ & 7 & 40 & 6 \\
\hline$B$ bifidum & ATCC $29521^{\top}$ & 22 & 19 & 100 & 27 & 13 \\
\hline$B$ breve & ATCC $15700^{\top}$ & & & & 100 & 13 \\
\hline$B$ animalis & $\begin{array}{l}\text { NCFB } 2242^{\top} \\
\text { ATCC } 25527^{\top}\end{array}$ & 5 & 0 & 3 & 3 & $\begin{array}{l}100 \\
127\end{array}$ \\
\hline$B L$ & & 127 & 87 & 13 & 40 & 14 \\
\hline $\mathrm{BI}$ & & 9 & 8 & 5 & 8 & 115 \\
\hline BB & & 10 & 8 & 7 & 6 & 108 \\
\hline
\end{tabular}

* Chaque valeur du coefficient d'hybridation (\% d'homologie) est la moyenne de 2 mesures indépendantes.

Each value of the hybridization is the average of 2 independent measurements.

souche BL hybride aussi fortement $(87 \%)$ avec la souche $B$ infantis DSM $20088^{\top}$ (tableau II). Cette étroite relation ADN-ADN est d'autre part confirmée par un pourcentage d'hybridation de $61 \%$ à $69 \%$ entre la souche type $B$ longum NCTC $11818^{\top}$ et la souche type $B$ infantis DSM $20088^{\top}$. Ces résultats sont en accord avec ceux rapportés dans la littérature (Bahaka et al,1993).

\section{L'espèce $B$ animalis}

Les pourcentages de réassociation obtenus entre la souche $B$ animalis NCFB $2242^{\top}, \mathrm{Bl}$ et $\mathrm{BB}$ sont tous supérieurs à $100 \%$, ce qui nous permet de conclure que $\mathrm{Bl}$ et $\mathrm{BB}$ appartiennent bien à l'espèce $B$ ani- malis. Cette étroite parenté des 3 souches est confirmée par des valeurs de $\Delta T \mathrm{~T}$ inférieures à $2,2^{\circ} \mathrm{C}$.

\section{Polymorphisme de restriction de l'ADN par électrophorèse en champs pulsés}

Selon Grimont et Grimont (1991) et Forbes et al (1991), l'ADN chromosomique digéré par une endonucléase de restriction et visualisé après électrophorèse en champs pulsés fournit une empreinte caractéristique utilisable pour la classification d'isolats. Cette technique permet d'établir le profil de restriction de l'ADN constitutif, et aussi de mesurer la taille du génome. Le nombre et 
la taille des fragments du génome des souches étudiées après hydrolyse par Xbal sont rassemblés dans le tableau III.

Les profils de restriction de $B$ infantis ATCC $15697^{\top}$ et $B$ infantis DSM $20088^{\top}$ sont similaires et se caractérisent par la présence de 18 fragments de taille variant de $13 \mathrm{~kb}$ à $204 \mathrm{~kb}$. Ils se distinguent des autres profils par un nombre plus important de fragments, notamment de faible taille.

La séparation des fragments d'ADN digéré par Xbal de la souche BL est diffuse, ce qui rend difficile la comparaison de leur profil. Cependant, la présence d'une bande de taille élevée $(280 \mathrm{~kb})$ permet de différencier la souche BL des autres souches. De plus BL se distingue des autres souches par une taille supérieure $(2,1 \mathrm{Mb})$. Toutefois, cette valeur ne semble pas seulement caractéristique de l'espèce $B$ longum puisque la taille du génome de $B$ breve a été également estimée à 2,1 Mb par Bourget et al (1992).

$B$ animalis NCFB $2242^{\top}$ se distingue des autres profils par un faible nombre de fragments (10) et surtout par l'absence de fragments de taille comprise entre 55 et $97 \mathrm{~kb}$.

Les profils des souches $B I$ et $B B$, identifiées à l'espèce $B$ animalis, sont très proches l'un de l'autre par la taille de leur génome (respectivement voisine de 1,1 et $1,5 \mathrm{Mb}$ ) et le nombre de fragments. lls se distinguent toutefois par la présence de fragments de

Table III. Analyse de restriction Xbal et taille du génome des souches de Bifidobacterium après électrophorèse en champs pulsés.

$\mathrm{Xbal}$ restriction analysis and genome size of Bifidobacterium strains after pulsed field gel electrophoresis.

$\begin{array}{cllll}\text { B infantis } & \text { B animalis } \\ \text { DMS 20088 } & \text { BTCC 15697 }\end{array}$

$\begin{array}{lrrrrrr}\text { Nombre de fragments } & 18 & 17 & 18 & 13 & 13 & 10 \\ \text { Taille du génome (en Mb) } & 1,3 & 1,4 & 2,1 & 1,1 & 1,5 & 1,1 \\ \text { Tailles des fragments } & 204 & 203 & 282 & 223 & 278 & 251 \\ \text { digérés par Xbal (en kb) } & 188 & 187 & 238 & 177 & 223 & 202 \\ & 142 & 142 & 215 & 162 & 209 & 167 \\ & 116 & 139 & 183 & 131 & 180 & 133 \\ & 100 & 117 & 174 & 89 & 167 & 102 \\ & 92 & 100 & 136 & 71 & 136 & 97 \\ & 83 & 91 & 127 & 58 & 91 & 55 \\ & 68 & 83 & 113 & 50 & 71 & 45 \\ & 59 & 67 & 108 & 39 & 59 & 38 \\ & 49 & 58 & 96 & 28 & 50 & 26 \\ & 41 & 48 & 88 & 23 & 40 & \\ & 37 & 41 & 73 & 19 & 31 & \\ & 31 & 37 & 67 & 15 & 23 & \\ & 23 & 31 & 54 & & & \\ & 21 & 21 & 46 & & & \\ & 18 & 18 & 43 & & & \end{array}$


taille de $278 \mathrm{~kb}$ et $209 \mathrm{~kb}$ chez la souche BB. Ces souches se différencient ainsi de la souche type par la présence de fragments de taille comprise entre 55 et $97 \mathrm{~kb}$. Ces profils permettent donc de distinguer les souches au sein de l'espèce $B$ animalis.

\section{CONCLUSION}

L'identification des espèces du genre Bifidobacterium employées dans les produits laitiers présente un intérêt essentiel pour évaluer les effets que ces micro-organismes pourraient avoir sur la santé de l'hôte. Or à ce jour, peu de méthodes de caractérisation, permettant de vérifier correctement les souches utilisées, sont facilement applicables dans un laboratoire industriel.

En vue d'obtenir un moyen de caractérisation plus discriminant, 3 souches pures de bifidobactéries provenant de ferments ont été identifiées et caractérisées par deux méthodes de taxonomie génomique.

L'hybridation ADN-ADN a été utilisée comme méthode de référence pour identifier l'espèce de ces bactéries. Elle a permis de confirmer une forte similitude génomique de la souche BL avec la souche type de l'espèce $B$ longum, tandis que les souches $\mathrm{Bl}$ et $\mathrm{BB}$ commercialisées comme appartenant respectivement aux espèces $B$ infantis et $B$ bifidum ont été identifiées à l'espèce $B$ animalis.

Si l'hybridation ADN-ADN permet de séparer les espèces, la comparaison des profils de restriction permet de différencier chacune des souches testées. De plus, cette technique a permis d'évaluer de manière préliminaire la taille globale de l'ADN chromosomique qui varie de 1,1 à 2,1 $\mathrm{Mb}$ selon les souches. La différence observée demanderait cependant à être vérifiée avec une autre enzyme de restriction. Cette méthode devrait permettre dans le domaine industriel de reconnaître et de suivre (sous forme d'empreintes génomiques) la présence de telle ou telle souche dans des populations mixtes.

\section{REMERCIEMENTS}

Cette étude fait partie du travail réalisé pour l'obtention d'un diplôme d'ingénieur CNAM en biologie industrielle et agro-alimentaire.

Nous remercions F Gavini pour l'envoi de certaines souches-type.

\section{RÉFÉRENCES}

Bahaka D, Neut C, Khattabi A, Monget D, Gavini F (1983) Phenotypic and genomic analyses of human strains belonging or related to Bifidobacterium longum, Bifidobacterium infantis, and Bifidobacterium breve. Int J Syst Bacteriol 43, 565-573

Bourget B, Simonet JM, Decaris B (1992) Estimation de la taille du génome, du nombre de loci ribosomiques et du contenu plasmidique chez Bifidobacterium breve. Ve reunion du Club des bactéries lactiques, CBL 92, Nancy, 2-9 septembre

Crosa JH, Brenner DJ, Falkow S (1973) Use of a singlestrand specific nuclease for analysis of bacterial and plasmid deoxyribonucleic acid homo- and heteroduplexes. J Bacteriol 115, 904-911

Forbes KJ, Bruce KD, Jordens ZJ, Ball A, Pennington TH (1991) Rapid methods in bacterial DNA fingerpriting. J Gen Microbiol 137, 2051-2058

Fuller R (1992) History and development of probiotics. In: Probiotics. The scientific basis (Fuller R, ed). Chapman \& Hall, London

Gavini F (1989) Bifidobacterium: Classification; aspect critique. In: Bifidobacterium et facteurs bifidogènes (Association pour la recherche sur les bifidobactéries et les bactéries anaérobies, éd). ID2 Communication, Paris, 47-53

Grimont PAD (1988) Use of DNA reassociation in bacterial classification. Can J Microbiol 34, 541-546

Grimont F, Grimont PAD (1991) DNA Fingerprinting. In: Nucleic acid techniques in bacterial systematics (Stackebrandt E, Goodfellow M, eds). John Wiley \& sons, Chichester

Peitersen N (1991) Probiotic starter cultures of food products. In: Les bactéries lactiques : actes du colloque LACTIC 1991 (Novel G, Le Querler JF, eds). Centre de publications de l'université de Caen

Tanskanen El, Tulloch DL, Hillier AJ, Davidson BE (1990) Pulsed-field gel electrophoresis of Smal digests of lactococcal genomic DNA, a novel method of strain identification. Appl Environ Microbiol 56, 3105-3111

Wayne LG, Brenner DJ, Colwell RR et al (1987) Report of the ad hoc committee on reconciliation of approaches to bacterial systematics. Int J Syst Bacteriol 37, 463-464 